\title{
Mid-term follow-up outcomes of 2-staged hybrid coronary revascularization compared with off-pump coronary artery bypass for patients with multivessel coronary artery disease
}

\author{
Song Wu, Yunpeng Ling, Yuanhao Fu, Lufeng Zhang, Hang Yang, Lijun Guo, Wei Gao, Feng Wan \\ Peking University Third Hospital, Beijing, China
}

Videosurgery Miniinv 2017; 12 (2): 178-185

DOI: https://doi.org/10.5114/wiitm.2017.66803

\begin{abstract}
Introduction: Two-staged hybrid coronary revascularization $(H C R)$ is a novel procedure in selected patients with multivessel coronary artery disease. However, few studies are available on the mid-term or long-term outcomes of this 2-staged procedure as compared to off-pump coronary artery bypass (OPCAB).

Aim: To compare in-hospital and mid-term follow-up outcomes of 2-staged HCR with OPCAB in patients with multivessel coronary artery disease.

Material and methods: The present retrospective study analyzed the data from 73 patients who underwent the 2-staged HCR (HCR group) during 2012-2014 at the heart center. Three hundred and eighty-three patients who underwent conventional OPCAB by the same surgeon were selected for the comparative analysis performed on in-hospital and mid-term follow-up outcomes between the two groups.

Results: No significant difference was observed in the preoperative outcome between the two groups. The HCR group had a shorter operation duration (152.9 $\pm 43.8 \mathrm{vs}$. $262.6 \pm 51.8 \mathrm{~min}, p<0.05)$, less bleeding (558.6 $\pm 441.3 \mathrm{vs} .1035 .5$ $\pm 613.3 \mathrm{ml}, p<0.05)$, shorter mechanical ventilation (9.4 $\pm 7.4 \mathrm{vs} .19 .0 \pm 18.3 \mathrm{~h}, p<0.05$ ), and less blood transfusion (12 (16.4\%) vs. 200 (52.2\%), $p<0.05)$. The mean follow-up duration was $25.0 \pm 9.6$ months in the HCR group and $22.8 \pm 10.6$ months in the OPCAB group. The incidence of major adverse cardiac or cerebrovascular events (MACCE) was similar in both groups (4 (5.5\%) vs. 10 (2.8\%), $p=0.408)$. The estimated 3-year survival was similar in both groups (log-rank $\left.\chi^{2}=1.041, p=0.308\right)$.

Conclusions: The 2-staged HCR is a safe and effective surgical procedure and may offer similar mid-term follow-up outcomes to OPCAB.
\end{abstract}

Key words: two-staged hybrid coronary revascularization, percutaneous coronary intervention, off-pump coronary artery bypass, minimally invasive direct coronary artery bypass graft surgery, follow-up outcomes.

\section{Introduction}

Coronary artery bypass graft (CABG) surgery remains the standard strategy to achieve complete revascularization in patients with multivessel coronary artery disease. The superiority of CABG over multivessel percutaneous coronary intervention $(\mathrm{PCl})$ with respect to cardiac death, myocardial in- farction, and long-term respite from repeated coronary revascularization has been proved by several studies and randomized trials [1]. Nowadays, a hybrid coronary revascularization (HCR) strategy has been accepted as the procedure of choice for coronary artery disease (CAD), combining the most effective therapeutic methods of cardiovascular surgery 
and interventional cardiology in order to perform a functionally complete revascularization [2]. With $\mathrm{HCR}$, minimally invasive direct coronary artery bypass graft surgery (MIDCABG) using a left internal mammary artery (LIMA) graft to the left anterior descending (LAD) coronary artery is combined with $\mathrm{PCI}$ to non-LAD targets. This approach capitalizes on the major strengths associated with CABG and PCI. The benefits of $C A B G$ are largely attributable to the LIMA-LAD graft owing to its long-term patency and freedom from atherosclerosis. The failure rates of saphenous vein grafts and the lower restenosis rates with drug-eluting stents (DES) have made the percutaneous treatment of non-LAD vessels ubiquitous and comparable to saphenous vein grafts [3-6].

The HCR can be performed either as a 1-stop or as a 2-staged procedure. The former implies concomitant $\mathrm{CABG}$ and $\mathrm{PCI}$ in a single operative suite, with $\mathrm{PCl}$ following the CABG almost instantly. The 2-staged procedure is defined as $\mathrm{PCl}$ and $\mathrm{CABG}$ performed in 2 different operative suites; the 2 procedures are separated by hours, days, or weeks, whereas the 1-stop refers to hybrid CABG/PCI performed in a hybrid suite in 1 setting, within minutes. Initially, the 1-stop HCR was considered to be a safe and feasible option with more acceptable mid-term clinical outcomes than CABG and $\mathrm{PCl}$ in selected patients with multivessel CAD [7-10]. However, few studies on the mid-term or long-term outcomes of the 2-staged procedure as compared to the OPCAB strategy are available.

\section{Aim}

In this study, we compared the in-hospital and mid-term clinical outcomes of the 2-staged HCR and OPCAB procedures at our hospital.

\section{Material and methods}

\section{Patient selection}

The relative angiographic indications for HCR include the presence of significant proximal LAD disease or left main equivalent that is amenable to LIMA-to-LAD bypass and non-LAD lesions that allow $\mathrm{PCl}$. The relative clinical contraindications for HCR include hemodynamic instability, previous cardiac or thoracic surgery, a severe lung disease with the inability to tolerate single-lung ventilation, and severe morbid obesity. The inclusion criteria for the 2-staged HCR were as follows: 1) unfavorable LAD for $\mathrm{PCl}$, unprotected left main CAD, and non-LAD lesions technically feasible for $\mathrm{PCI}$ with a DES; 2) limitations of traditional CABG, such as pre-existing organ dysfunction, heavily calcified proximal aorta, or lack of suitable graft conduits. The exclusion criteria for the 2-staged HCR included left subclavian artery and LIMA stenosis, buried intramyocardial LAD, need for a simultaneous operation, apparent congestive heart failure, hemodynamic instability, and other conditions rendering $\mathrm{PCl}$ unsuitable [8-10].

\section{Surgical procedure}

\section{Two-staged HCR procedure}

In the HCR group, the patients were anesthetized and intubated with double-lumen endotracheal tubes. Single-lung ventilation was employed to facilitate exposure for LIMA harvesting. The patients were positioned supine with a $30^{\circ}$ rotated decubitus towards the right using a rolled towel. External pads for emergency defibrillation were placed on the right anterior and left posterior sides of the chest wall. A small, left anterior thoracotomy incision $(5-7 \mathrm{~cm})$ was facilitated in the $4^{\text {th }}$ or $5^{\text {th }}$ intercostal space, according to the preoperative chest $\mathrm{X}$-ray and coronary angiography. Typically, LIMA harvesting was initiated from superior to inferior due to the absence of transthoracic muscle at the superior border. Fat tissue was removed with a pedicle in order to identify the LIMA. The LIMA was harvested superior to the $1^{\text {st }}$ rib (close to the left subclavian vein) and inferior to the upper portion of the $5^{\text {th }}$ or $6^{\text {th }}$ costal cartilage. After LIMA preparation and dilation with papaverine solution, blood flow from the LIMA was confirmed. A pericardial incision exposed and stabilized the LAD. A shunt was routinely used after coronary arteriotomy instead of target vessel occlusion. A continuous running suture technique was used to perform the coronary anastomosis. In all the HCR cases, the LIMA-LAD anastomosis was performed first as part of the staged procedure on separate days. Postoperatively, $\mathrm{PCl}$ for the remaining non-LAD lesions was performed only after documenting a patient LIMA-LAD anastomosis in the catheterization laboratory, commonly 3-5 days after the surgical procedure. We emphasized that heparin should be administered as soon as possible after surgery if chest tube drainage was less than $50 \mathrm{ml} / \mathrm{h}$ for $6 \mathrm{~h}$ after ensuring the absence of bleeding com- 
plications. On the next day, dual antiplatelet therapy (DAPT) was begun, coupled with aspirin $(100 \mathrm{mg} /$ day) and clopidogrel (75 mg/day). In the PCl-first approach in 2-staged HCR, DAPT was typically commenced ahead of the $\mathrm{PCl}$ procedure and continued during $C A B G$. After the procedure, all patients were subjected to continuous aspirin therapy (100 mg/ day for a lifetime), and clopidogrel (75 mg/day) was prescribed for 12 months following DES implantation.

\section{OPCAB procedure}

In the OPCAB group, coronary revascularization was performed in 383 patients via sternotomy using pedicled LIMA for LAD artery grafting. The procedures were performed in all patients under general anesthesia and tracheal intubation. The LIMA and saphenous vein conduits were harvested under direct vision. The distal anastomoses of the LIMA to LAD and saphenous conduit to non-LAD target grafts were performed using a stabilizer on the beating heart. A suction-based epicardial stabilizer and an intracoronary shunt were routinely used. The saphenous veins were used for non-LAD vessel grafting. The proximal anastomoses were accomplished with a partial occluding aortic clamp [11, 12].

\section{Outcomes}

The primary endpoint of the present study was to evaluate the safety and feasibility of the 2-staged $\mathrm{HCR}$ and compare the in-hospital outcomes between the 2 groups. The secondary endpoints constituted the incidence of major adverse cardiac or cerebrovascular events (MACCE), as well as a composite of death, myocardial infarction, neurologic event, and target lesion or vessel repeat revascularization during the follow-up period. All patients were required to return for a follow-up visit every 4 months after discharge. Myocardial perfusion imaging was recommended for patients without ischemic symptoms. Angiography was performed if noninvasive diagnostic tests indicated a sign of ischemia. Both myocardial perfusion and angiography were strongly recommended for patients with recurrence of ischemic symptoms [7].

\section{Statistical analysis}

The continuous variables are presented as the mean \pm SD and the categorical variables as frequen- cies and percentages. The categorical variables were compared between the two groups using the $\chi^{2}$ or Fisher's exact test. For continuous variables, Student's $t$-test was used to estimate the differences in a normal distribution. The MACCE were analyzed using the Kaplan-Meier method and the log-rank test, and the difference with a $95 \%$ confidence interval was calculated. A $p$-value $<0.05$ was considered statistically significant. All statistical analyses were performed using SPSS software version 17.0 (SPSS Inc., Chicago, IL, USA).

\section{Results}

\section{Patient characteristics}

After propensity score matching, the baseline characteristics were similar in the two groups (Table I).

\section{Perioperative outcomes}

In the HCR group, 73 (100\%) patients successfully underwent 2-staged hybrid procedures. They received a LIMA-LAD graft and $P C I$ with DES for nonLAD lesions. The mean number of DES implanted in a patient was $1.6 \pm 0.7$, and the mean number of revascularizations in a patient was $2.6 \pm 0.5$. The time interval from MIDCABG to $\mathrm{PCl}$ was $5.3 \pm 2.9$ days in the study. In the OPCAB group, 383 patients received a LIMA-LAD graft for LAD and a saphenous vein graft for non-LAD targets. These patients underwent offpump surgery, and the mean number of revascularizations in the patients was $2.7 \pm 0.5$ without a significant difference between the two groups $(2.6 \pm 0.5$ vs. $2.7 \pm 0.5, p>0.05)$. The lesser invasiveness of HCR could offer superior perioperative outcomes, including reduced duration of operation $(152.9 \pm 43.8 \mathrm{vs}$. $262.6 \pm 51.8 \mathrm{~min}, p<0.05)$, intubation $(10.5 \pm 13.0 \mathrm{vs}$. $30.5 \pm 61.3 \mathrm{~h}, p<0.05)$, and ICU stay $(38.2 \pm 27.0 \mathrm{vs.}$ $69.0 \pm 185.9 \mathrm{~h}, p<0.05)$, as well as less transfusion (12 (16.4\%) vs. 200 (52.2\%), $p<0.05)$ than OPCAB. It does not increase either the risk of perioperative myocardial infarction (0 (0\%) vs. $1(0.3 \%), p>0.05)$ or the postoperative renal dysfunction (74.4 \pm 11.9 vs. $75.8 \pm 12.7 \mu \mathrm{mol} / \mathrm{l}, p>0.05)$. No significant difference was observed between the two groups with respect to re-exploration for bleeding (1 (1.4\%) vs. $6(1.6 \%), p>0.05)$ or total drainage $(558.6 \pm 441.3$ vs. $1035.5 \pm 613.3 \mathrm{ml}, p<0.05)$. The mean postoperative incision pain VAS scores were higher for HCR than OPCAB $(6.5 \pm 1.71$ vs. $3.3 \pm 0.9, p=0.001)$ (Table II). 
Table I. Demographic and clinical baseline characteristics

\begin{tabular}{|c|c|c|c|c|}
\hline Variables & HCR group & OPCAB group & $t / \chi^{2}$ & $P$-value \\
\hline Age [years] & $61.1 \pm 10.7$ & $63.1 \pm 8.9$ & -1.757 & 0.08 \\
\hline Male, $n(\%)$ & $50(68.5)$ & $286(74.7)$ & 1.208 & 0.272 \\
\hline $\mathrm{BMI}\left[\mathrm{kg} / \mathrm{m}^{2}\right]$ & $25.5 \pm 2.7$ & $25.1 \pm 3.3$ & 0.948 & 0.344 \\
\hline \multicolumn{5}{|l|}{ CCS, $n(\%):$} \\
\hline 1 & $17(23.2)$ & $84(21.9)$ & 1.071 & 0.784 \\
\hline II & 37 (50.7) & $177(46.2)$ & & \\
\hline III & $17(23.2)$ & $106(27.7)$ & & \\
\hline IV & $2(2.7)$ & $16(4.2)$ & & \\
\hline \multicolumn{5}{|l|}{ NYHA, $n(\%):$} \\
\hline I & $16(21.9)$ & $110(28.7)$ & 2.594 & 0.459 \\
\hline$\|$ & $44(60.3)$ & $216(56.4)$ & & \\
\hline III & $11(15.1)$ & $53(13.8)$ & & \\
\hline IV & $2(2.7)$ & $4(1.0)$ & & \\
\hline Previous $\mathrm{PCl}, n(\%)$ & $7(9.6)$ & $24(6.3)$ & 1.068 & 0.301 \\
\hline Previous myocardial infarction, $n(\%)$ & $10(13.7)$ & $73(19.1)$ & 1.184 & 0.277 \\
\hline Current smoker, $n(\%)$ & $35(47.9)$ & $209(54.6)$ & 1.081 & 0.298 \\
\hline Diabetes mellitus, $n$ (\%) & $26(35.6)$ & $134(35)$ & 0.011 & 0.918 \\
\hline Hypertension, $n$ (\%) & $46(63.0)$ & $212(55.4)$ & 1.465 & 0.226 \\
\hline Hypercholesterolemia, $n$ (\%) & $17(23.3)$ & $56(14.6)$ & 3.425 & 0.064 \\
\hline COPD, $n(\%)$ & $2(2.7)$ & $6(1.6)$ & 0.490 & 0.484 \\
\hline Peripheral arterial disease, $n$ (\%) & $1(1.4)$ & $15(3.9)$ & 1.174 & 0.278 \\
\hline Creatinine level [ $\mathrm{mmol} / \mathrm{l}]$ & $75.2 \pm 20.0$ & $71.5 \pm 22.5$ & 1.306 & 0.192 \\
\hline Total cholesterol level [ $\mu \mathrm{mol} / \mathrm{l}]$ & $4.2 \pm 1.1$ & $4.7 \pm 1.0$ & -3.556 & $<0.001$ \\
\hline Previous cerebrovascular accident, $n$ (\%) & $12(16.4)$ & $55(14.4)$ & 0.211 & 0.646 \\
\hline $\mathrm{LM}, n(\%)$ & $14(19.2)$ & $71(18.5)$ & 0.017 & 0.898 \\
\hline LVEF (\%) & $60.0 \pm 12.0$ & $59.3 \pm 11.5$ & 0.451 & 0.652 \\
\hline LVEDD $[\mathrm{mm}]$ & $50.4 \pm 6.8$ & $53.6 \pm 42.8$ & -0.653 & 0.514 \\
\hline EuroSCORE & $3.6 \pm 2.4$ & $3.6 \pm 1.7$ & -0.375 & 0.708 \\
\hline
\end{tabular}

BMI - body mass index, CCS - Canadian Cardiovascular Society, COPD - chronic obstructive pulmonary disease, EuroSCORE - European System for Cardiac Operative Risk Evaluation, LVEDD - left ventricular end-diastolic dimension, LVEF - left ventricular ejection fraction, NYHA - New York Heart Association.

\section{Mid-term outcomes}

The follow-up information and hospital records were completely reviewed by December 2015, in the HCR group $(73 / 73,100 \%)$ and the OPCAB group (360/383, 94.0\%). The mean follow-up was $25.0 \pm 9.6$ months in the HCR group and $22.8 \pm 10.6$ months in the OPCAB group. At a mean follow-up of 2 years, the actual survival rates in the HCR and OPCAB groups were $98.6 \%$ and $99.7 \%$, respectively. The survival curves of the two groups showed no significant difference (log-rank $p=0.308)$. The mid-term follow-up 
Table II. In-hospital outcomes of patients according to the study groups

\begin{tabular}{|c|c|c|c|c|}
\hline Variable & HCR group & OPCAB group & $t / \chi^{2}$ & $P$-value \\
\hline Operation time [min] & $152.9 \pm 43.8$ & $262.6 \pm 51.8$ & -16.993 & $<0.001$ \\
\hline No. of revascularizations $(n)$ & $2.6 \pm 0.5$ & $2.7 \pm 0.5$ & -1.644 & 0.101 \\
\hline Re-exploration for bleeding, $n$ (\%) & $1(1.4)$ & $6(1.6)$ & & $1.000^{*}$ \\
\hline Myocardial infarction, $n$ (\%) & $0(0)$ & $1(0.3)$ & & $1.000^{*}$ \\
\hline Neurologic event, $n(\%)$ & $0(0)$ & $1(0.3)$ & & $1.000^{*}$ \\
\hline New-onset atrial fibrillation, $n(\%)$ & $1(1.4)$ & $15(3.9)$ & 0.543 & 0.46 \\
\hline Total drainage $[\mathrm{ml}]$ & $558.6 \pm 441.3$ & $1035.5 \pm 613.3$ & -6.336 & $<0.001$ \\
\hline Total transfusion of RBC, $n(\%)$ & $12(16.4)$ & $200(52.2)$ & 31.555 & $<0.001$ \\
\hline Creatinine level $[\mu \mathrm{mol} / \mathrm{l}]$ & $74.4 \pm 11.9$ & $75.8 \pm 12.7$ & -0.881 & 0.379 \\
\hline cTnl $[\mu \mathrm{g} / \mathrm{l}])$ & $17.5 \pm 3.4$ & $17.0 \pm 4.0$ & 0.996 & 0.320 \\
\hline Postoperative ventilation [h] & $9.4 \pm 7.4$ & $19.0 \pm 18.3$ & -4.391 & $<0.001$ \\
\hline Time from MIDCABG to PCI [days] & $5.3 \pm 2.9$ & - & - & NA \\
\hline No. of stents used (n) & $1.6 \pm 0.7$ & - & - & NA \\
\hline ICU length of stay [h] & $31.6 \pm 17.0$ & $45.7 \pm 37.0$ & -3.195 & 0.001 \\
\hline In-hospital stay [days] & $18.4 \pm 7.9$ & $20.3 \pm 13.9$ & -1.150 & 0.251 \\
\hline $\begin{array}{l}\text { Mean postoperative incision pain VAS } \\
\text { scores }\end{array}$ & $6.5 \pm 1.71$ & $3.3 \pm 0.95$ & 5.16 & 0.001 \\
\hline $\begin{array}{l}\text { Maximum postoperative incision pain } \\
\text { VAS scores }\end{array}$ & 9 & 5 & - & NA \\
\hline
\end{tabular}

ICU - intensive care unit, MIDCABG - minimally invasive direct coronary artery bypass graft surgery, NA - not applicable, $P C I$ - percutaneous coronary intervention, CTnI - cardiac troponin I, VAS scores - visual analog scale scores ( 0 - no pain to 10 - worst pain ever experienced), "Fisher's test.

Table III. Mid-term follow-up outcomes

\begin{tabular}{|c|c|c|c|c|}
\hline Variable & HCR group & OPCAB group & $t / \chi^{2}$ & $P$-value \\
\hline Death, $n(\%)$ & $1(1.4)$ & $1(0.3)$ & & $0.309^{*}$ \\
\hline Myocardial infarction, $n(\%)$ & $3(4.1)$ & $8(2.2)$ & 0.873 & 0.598 \\
\hline Neurologic event, $n$ (\%) & $0(0)$ & $1(0.3)$ & & $1.000^{*}$ \\
\hline Repeat revascularization, $n$ (\%) & $1(1.4)$ & $6(1.7)$ & 0.000 & 1.000 \\
\hline Any MACCE, $n(\%)$ & $5(6.8)$ & $16(4.4)$ & 1.416 & 0.269 \\
\hline Follow-up time [months] & $25.0 \pm 9.6$ & $22.8 \pm 10.6$ & 1.693 & 0.091 \\
\hline Follow-up rate, $n(\%)$ & $73(100)$ & $360(94.0)$ & & \\
\hline Survival curves & & & 1.041 & 0.308 \\
\hline
\end{tabular}

${ }^{*}$ Fisher's test, MACCE - major adverse cardiac or cerebrovascular events.

results did not show any difference in mortality $(1.4 \%$ vs. $0.3 \%, p=0.210$ ), recurrent myocardial ischemia (4.1\% vs. $2.2 \%, p=0.350)$, repeat revascularization (1.4\% vs. $1.7 \%, p=0.855)$, neurological events (0\% vs. $0.3 \%, p=0.652)$, or the cumulative MACCE rate $(5.5 \%$ vs. $2.8 \%, p=0.269)$ in the HCR group as compared to the OPCAB group. One patient in the HCR group underwent repeat revascularization with $D E S$, and 6 patients in the OPCAB group received repeat revascularization with re-CABG (Table III, Figures 1 and 2). 


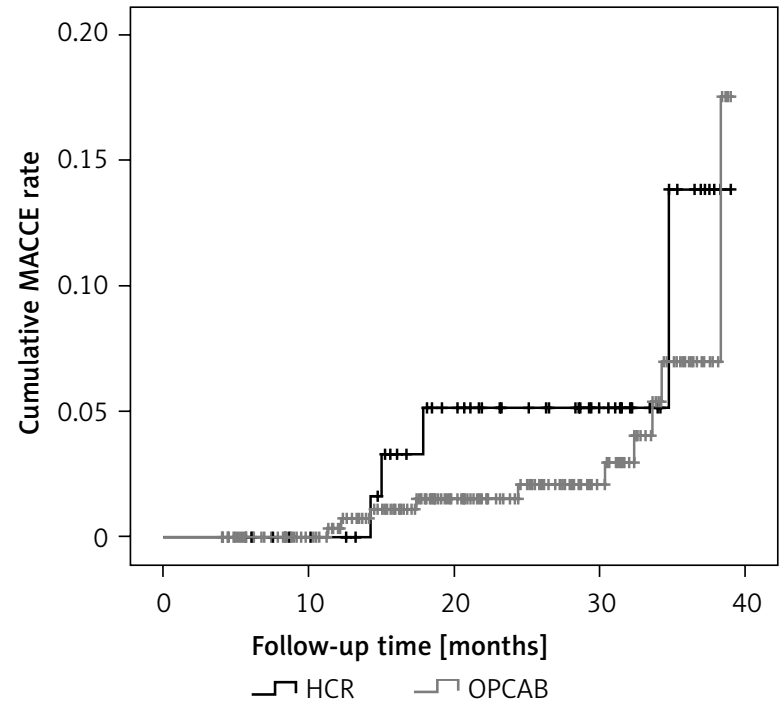

Figure 1. Cumulative MACCE rate in HCR and OPCAB groups. The cumulative MACCE rate in the HCR group $(5.5 \%)$ was similar to that in the OPCAB group $(2.8 \% ; p=0.269)$

\section{Discussion}

Presently, hybrid revascularization is being considered as a valid alternative to the traditional surgical strategy, fulfilling the prerequisites of both patients and cardiologists for less invasive treatments. 1-stop HCR has been proven as a safe and feasible option with acceptable mid-term clinical outcomes compared to $\mathrm{CABG}$ and $\mathrm{PCl}$ in selected patients with multivessel CAD [6, 7]. However, it is impossible to establish expensive hybrid operating rooms in many developing countries. The basic national conditions govern the imbalance of the regional economic development in China. Thus, several medical institutions do not possess the economic strength to build self-sufficient hybrid operating rooms. The 2-staged HCR procedures can offer a more realistic alternative to such institutions because 2-staged HCR procedures are more frequently employed than the 1-stop HCR procedures in China. Furthermore, only a few studies on the mid-term or long-term outcomes of this 2-staged strategy are available. Thus, in this study, we compared the in-hospital and mid-term follow-up outcomes of 2-staged HCR with OPCAB in patients with multivessel CAD to evaluate the security, reliability, and applicability of the procedure. In the 2-staged approach, the optimal order - PCI first vs. CABG first - is debated because each approach has advantages and disadvantages. Nevertheless,

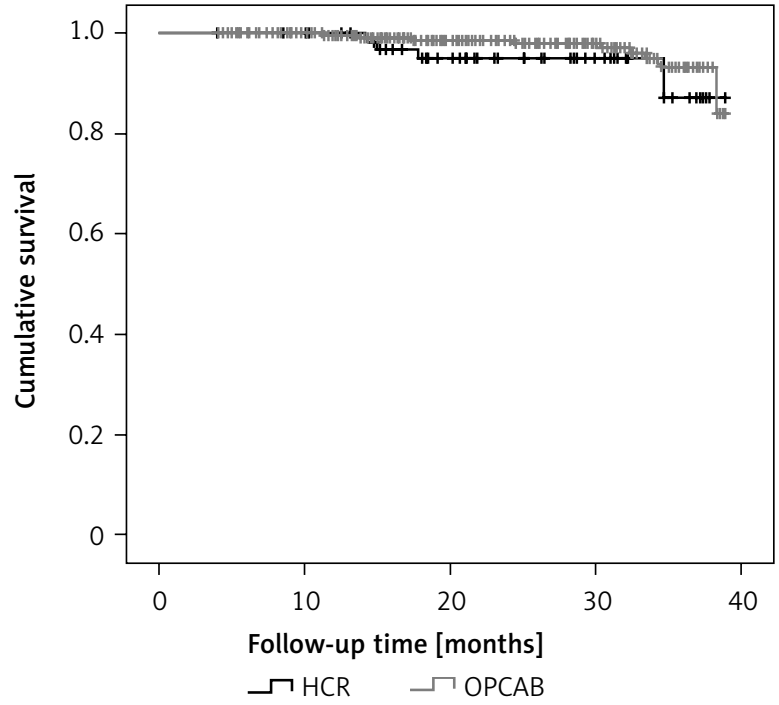

Figure 2. Kaplan-Meier 3-year survival estimates according to HCR or OPCAB $(p=0.308)$

these decisions should be guided by patient characteristics, operator skill/expertise, and available facilities. The American College of Cardiology Foundation/ American Heart Association guidelines favors CABG first $[13,14]$. Therefore, we adopted the strategy of CABG first, followed by $\mathrm{PCl}$ after a few days.

In order to obtain maximum benefits of the advantages of HCR, we developed a relatively positive procedure of antiplatelet management to balance the risk of perioperative bleeding with stent thrombosis [11, 12]. This antiplatelet management allowed the surgical step of 2-staged HCR to be performed with acceptable bleeding risk. In the majority of cases of the MIDCABG-first approach in 2-staged HCR, MIDCABG was carried out with aspirin $(100 \mathrm{mg}$ / day). Heparin was administered after surgery if the chest tube drainage was $<50 \mathrm{ml} / \mathrm{h}$ or $6 \mathrm{~h}$ after ensuring that no bleeding complications had occurred. The DAPT would begin with aspirin (100 mg/day) and clopidogrel (75 mg/day) on the next day. In the PCl-first approach in 2-staged HCR, DAPT was typically commenced ahead of the $\mathrm{PCl}$ procedure and was uninterrupted during MIDCABG. The results of this study showed that the relatively active anticoagulation strategy does not increase the risk of bleeding. Also, no significant differences were observed between the two groups in re-exploration for bleeding $(1(1.4 \%)$ vs. $6(1.6 \%), p>0.05)$. The lesser invasiveness of HCR could reduce the total transfusion of RBC (12 (16.4\%) vs. 200 (52.2\%), $p<0.05)$ and 
total drainage $(558.6 \pm 441.3$ vs. $1035.5 \pm 613.3 \mathrm{ml}$, $p<0.05)$ compared to OPCAB. Thus, this technology would undoubtedly have high clinical and social value because of the current shortage of blood supply. The 2-staged HCR technique could also offer other superior perioperative outcomes, including reduced lengths of operation time (152.9 \pm 43.8 vs. 262.6 $\pm 51.8 \mathrm{~min}, p<0.05)$, intubation $(10.5 \pm 13.0$ vs. 30.5 $\pm 61.3 \mathrm{~h}, p<0.05)$, and ICU stay (38.2 \pm 27.0 vs. 69.0 $\pm 185.9 \mathrm{~h}, p<0.05)$ compared to OPCAB. The time interval from MIDCABG to $\mathrm{PCl}$ was $5.3 \pm 2.9$ days in the 2-staged HCR group. At our center, the order and timing of the surgical and percutaneous interventional procedures were determined by the coronary anatomy of the patient and a joint decision between the surgeon and the interventional cardiologist. The interval of 3-5 days between procedures did not significantly prolong the patient's hospital stay (18.4 \pm 7.9 vs. $20.3 \pm 13.9$ days, $p>0.05$ ). The mean postoperative incision pain VAS scores were higher in the HCR vs. OPCAB group $(6.5 \pm 1.71$ vs. $3.3 \pm 0.9$, $p=0.001)$. The left anterior thoracotomy pain in the HCR group may originate from numerous sources, including the skin incision and deeper tissue injuries, thoracostomy tubes, costovertebral joint disruption, and also fractures of the ribs [15].

Although currently $\mathrm{PCl}$ therapy is the preferred method, CABG technology has been considered as an important treatment approach in high-risk patients with multivessel CAD. The benefits of CABG are largely attributable to the LIMA-LAD graft owing to its long-term patency and freedom from atherosclerosis [14-16]. In this study, all patients in the HCR group received LIMA-LAD grafts and $\mathrm{PCI}$ with DES for non-LAD lesions; the mean number of stents implanted in a patient was 1.9, and that of revascularizations was $2.6 \pm 0.5$. In the OPCAB group, the mean number of revascularizations in a patient was $2.7 \pm 0.5$, and all patients received LIMA-LAD grafts. No significant difference was recorded between the two groups in the number of revascularizations (2.6 \pm 0.5 vs. $2.7 \pm 0.5, p>0.05$ ), risk of perioperative myocardial infarction (0 (0\%) vs. $1(0.3 \%), p>0.05)$, or postoperative renal dysfunction $(74.4 \pm 11.9$ vs. 75.8 $\pm 12.7 \mu \mathrm{mol} / \mathrm{l}, p>0.05$ ). Patients who underwent the 2-staged HCR technique achieved complete coronary revascularization with outcomes equivalent to those for OPCAB. Some experts considered that 2 teams, double costs, longer hospital stays, logistical challenges, and potential risks were involved in 2-staged
HCR procedures [16-21]. Owing to the close cooperation between the surgeon and the interventional cardiologists at our center, we performed the 2-staged HCR as a planned tailored strategy based on the patient's coronary lesions for optimal results. Thus, the 2-staged HCR procedure could be completed by one 2-staged HCR medical team, the patients were admitted once, and one HCR procedure was performed in two stages at one cardiovascular center, thereby avoiding the potential risks. The cost factor may include the financial resources expended on the construction of a cardiac hybrid operating room as well as training of the personnel. The construction of such rooms typically requires the conversion of at least 2 standard operating rooms. Thus, we speculate that the 2-staged HCR approach may be preferable for many patients in developing countries.

Previous studies showed that the 1-stop HCR technique had satisfactory results in the revascularization of multiple vessel diseases. The incidence of postoperative MACCE of 1-stop HCR was significantly lower than that of $\mathrm{PCl}$ after 1-3 years of follow-up, and the results were similar to those of the CABG group [7, 21-23]. The 2-staged HCR technique was in agreement with the current economic condition of the country. The long-term survival rate and the incidence of MACCE of the 2-staged HCR are still under further investigation. Some studies showed that the 1-year follow-up incidence of MACCE in 2-staged HCR was lower than CABG or similar; no difference was observed between 1-stop and 2-staged HCR. In our study, the mid-term follow-up (25.0 \pm 9.6 months) results showed no difference in mortality $(1.4 \%$ vs. $0.3 \%, p=0.210)$, recurrent myocardial ischemia ( $4.1 \%$ vs. $2.2 \%, p=0.350$ ), repeat revascularization (1.4\% vs. $1.7 \%, p=0.855)$, neurologic events ( $0 \%$ vs. $0.3 \%, p=0.652$ ), or MACCE ( $5.5 \%$ vs. $2.8 \%, p=0.269$ ) when comparing the 2-staged HCR group with the OPCAB group. The survival curves of the two groups did not reveal any significant difference between the two groups (log-rank test, $p=0.308$ ). Based on the mid-term follow-up results, the 2-staged HCR can be deemed an effective and safe treatment method. It may be used as one of the treatment strategies for patients with multiple CAD, especially those with high-risk factors and poor economic conditions. Without an excessive financial burden, the patient may enjoy the same medical service, and the 2-staged HCR would have its development space. 


\section{Conclusions}

Our clinical findings suggested that 2-staged HCR was a safe and effective surgical approach with little trauma and rapid postoperative recovery. As compared to OPCAB, 2-staged HCR was a safe treatment method with respect to in-hospital outcomes and mid-term follow-up symptomatic relief, major adverse cardiac or cerebrovascular events, and survival benefit.

This is a retrospective case-control study from a single center and a single surgeon. Follow-up angiograms were only performed in symptomatic patients. The sample size was small. Thus, a prospective study and longer follow-up duration are imperative in a large multicenter sample randomized controlled trial.

\section{Conflict of interest}

The authors declare no conflict of interest.

\section{References}

1. Wijns W, Kolh P, Danchin N, et al. Guidelines on myocardial revascularization. Task Force on Myocardial Revascularization of the European Society of Cardiology (ESC) and the European Association for Cardio-Thoracic Surgery (EACTS); European Association for Percutaneous Cardiovascular Interventions (EAPCI). Eur Heart J 2010; 31: 2501-55.

2. Angelini GD, Wilde P, Salerno TA, et al. Integrated left small thoracotomy and angioplasty for multivessel coronary artery revascularization. Lancet 1996; 347: 757-8.

3. Loop FD, Lytle BW, Cosgrove DM, et al. Influence of the internal-mammary-artery graft on 10-year survival and other cardiac events. N Engl J Med 1986; 314: 1-6.

4. Harskamp RE, Lopes RD, Baisden CE, de Winter RJ, Alexander $\mathrm{JH}$. Saphenous vein graft failure after coronary artery bypass surgery: pathophysiology, management, and future directions. Ann Surg 2013; 257: 824-33.

5. Byrne JG, Leacche M, Vaughan DE, Zhao DX. Hybrid cardiovas cular procedures. JACC Cardiovasc Interv 2008; 1: 459-68.

6. Bachinsky WB, Abdelsalam M, Boga G, et al. Comparative study of same sitting hybrid coronary artery revascularization versus off-pump coronary artery bypass in multivessel coronary artery disease. J Interv Cardiol 2012; 25: 460-8.

7. Shen L, Hu S, Wang H, et al. One-stop hybrid coronary revascularization versus coronary artery bypass grafting and percutaneous coronary intervention for the treatment of multivessel coronary artery disease: 3 -year follow-up results from a single institution. J Am Coll Cardiol 2013; 61: 2525-33.

8. Avgerinos DV, Charitakis K. Hybrid coronary revascularization: present and future. Hellenic J Cardiol 2015; 56: 193-6.

9. Verhaegh AJ, Accord RE, van Garsse L, Maessen JG. Hybrid coronary revascularization as a safe, feasible, and viable alternative to conventional coronary artery bypass grafting: what is the current evidence? Minim Invasive Surg 2013; 2013: 142616.

10. Harskamp RE, Zheng Z, Alexander JH, et al. Status quo of hybrid coronary revascularization for multi-vessel coronary artery disease. Ann Thorac Surg 2013; 96: 2268-77.

11. Ling Y, Bao L, Yang W, et al. Minimally invasive direct coronary artery bypass grafting with an improved rib spreader and a new-shaped cardiac stabilizer: results of 200 consecutive cases in a single institution. BMC Cardiovasc Disord 2016; 16: 42.

12. Zhang L, Cui Z, Song Z, et al. Minimally invasive direct coronary artery bypass for left anterior descending artery revascularization - analysis of 300 cases. Videosurgery Miniinv 2016; 10 : 548-54.

13. Comez M, Celik M, Dostbil A, et al. The effect of pre-emptive intravenous Dexketoprofen + thoracal epidural analgesia on the chronic post-thoracotomy pain. Int J Clin Exp Med 2015; 8: 8101-7.

14. Bachinsky WB, Abdelsalam M, Boga G, et al. Comparative study of same sitting hybrid coronary artery revascularization versus off-pump coronary artery bypass in multivessel coronary artery disease. J Interv Cardiol 2012; 25: 460-8.

15. Ochroch EA, Gottschalk A. Impact of acute pain and its management for thoracic surgical patients. Thorac Surg Clin 2005; 15: 105-21.

16. Delhaye C, Sudre A, Lemesle G, et al. Hybrid revascularization, comprising coronary artery bypass graft with exclusive arterial conduits followed by early drug-eluting stent implantation, in multivessel coronary artery disease. Arch Cardiovasc Dis 2010; 103: 502-11.

17. Halkos ME, Vassiliades TA, Douglas JS, et al. Hybrid coronary revascularization versus off-pump coronary artery bypass grafting for the treatment of multivessel coronary artery disease. Ann Thorac Surg 2011; 92: 1695-701; discussion 1701-2.

18. Serruys PW, Morice MC, Kappetein AP, et al.; SYNTAX Investigators. Percutaneous coronary intervention versus coronary-artery bypass grafting for severe coronary artery disease. N Engl J Med 2009; 360: 961-72.

19. Kon ZN, Brown EN, Tran R, et al. Simultaneous hybrid coronary revascularization reduces postoperative morbidity compared with results from conventional off-pump coronary artery bypass. J Thorac Cardiovasc Surg 2008; 135: 367-75.

20. Zhao DX, Leacche M, Balaguer JM, et al. Routine intraoperative completion angiography after coronary artery bypass grafting and 1-stop hybrid revascularization results from a fully integrated hybrid catheterization laboratory/operating room. J Am Coll Cardiol 2009; 53: 232-41.

21. Holzhey DM, Jacobs S, Mochalski M, et al. Minimally invasive hybrid coronary artery revascularization. Ann Thorac Surg 2008; 86: 1856-60.

22. Adams C, Burns DJ, Chu MW, et al. Single-stage hybrid coronary revascularization with long-term follow-up. Eur J Cardiothorac Surg 2014; 45: 438-42; discussion 442-3.

23. Repossini A, Tespili M, Saino A, et al. Hybrid coronary revascularization in 100 patients with multivessel coronary disease. Ann Thorac Surg 2014; 98: 574-80; discussion 580-1.

Received: 4.02.2017, accepted: 8.03.2017. 